\title{
Physical parameters of a mid-latitude streamer during the declining phase of the solar cycle
}

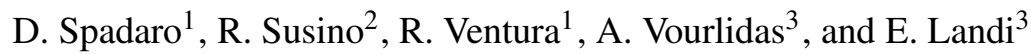 \\ 1 INAF-Osservatorio Astrofisico di Catania, via S. Sofia 78, 95123 Catania, Italy \\ e-mail: [dspadaro; rventura] @oact.inaf.it \\ 2 Dipartimento di Fisica e Astronomia - Sezione Astrofisica, Università di Catania, via S. Sofia 78, 95123 Catania, Italy \\ e-mail: sur@oact.inaf.it \\ 3 Naval Research Laboratory, 4555 Overlook Avenue, SW, Washington, DC 20375-5320, USA \\ e-mail: [vourlidas; landi]@nrl.navy.mil
}

Received 14 May 2007 / Accepted 7 September 2007

\begin{abstract}
Context. Investigating the physical properties of solar coronal streamers is important for understanding their role in the global magnetic structure of the extended solar atmosphere, as well as in the generation of the slow solar wind.

Aims. We hope to contribute as completely as possible to the ongoing SOHO instruments campaign devoted to the study of the physical characteristics of coronal streamers at various heliocentric distances.

Methods. We analyzed ultraviolet HI Ly $\alpha$ and O VI resonance doublet lines observed by UVCS/SOHO in a narrow, mid-latitude streamer structure along different lines of sight during a week in May 2004 and made nearly simultaneous white-light polarized brightness measurements from the LASCO/SOHO C2 coronagraph.

Results. Electron densities and temperatures, HI and O VI kinetic temperatures, and outflow velocities were derived from the line intensities and widths, as well as from the $\mathrm{O}$ VI line intensity ratio in the $1.6-5 R_{\odot}$ range of heights, limited to the central region of the streamer. To our knowledge, the $\mathrm{H}$ I outflow velocities obtained in this work are the first ones determined inside a streamer structure. They are significantly lower than those of the O VI ions. This, together with the O VI kinetic temperatures that are much higher than the H I ones, suggest that the absorption of Alfvén waves at the ion cyclotron frequency might also occur inside streamers.

Conclusions. In comparison with other streamers described in the literature, the structure examined in this work generally exhibits lower electron density and neutral hydrogen kinetic temperature. Conversely, the O vI kinetic temperature and outflow velocity radial profiles are consistent with the results for the other examined streamers.
\end{abstract}

Key words. Sun: corona - Sun: solar wind - Sun: UV radiation

\section{Introduction}

Streamers can be described as collections of open and closed magnetic flux tubes that give rise to elongated, high-density, helmet, or fan-shaped structures extending from the Sun into interplanetary space (see, e.g., Noci et al. 1997; Kohl et al. 2006). During solar maximum, they can be found at almost every latitude, while they are located during minimum around the solar equator, forming the so-called streamer belt.

Investigating the physical properties of solar coronal streamers as completely as possible is important for understanding the role that they play both in the global magnetic structure of the extended solar atmosphere (see, e.g., Low 2001) and in the generation of the largely variable, slow-speed solar wind believed to originate within such features. Many recent works have focused on this topic (e.g., Marsch 1997; Noci et al. 1997; Raymond et al. 1997; Habbal et al. 1997; Woo \& Martin 1997), but - at present - a firm identification of the slow wind-source regions inside streamers is still an unresolved issue.

Up to about a decade ago, most of the information on streamers came from visible-continuum eclipse observations, from which profiles of density vs. height have been derived at different times during the solar activity cycle. Following the launch of SOHO (SOlar and Heliospheric Observatory) satellite in 1995 (Domingo et al. 1995), new information on streamer properties has become available, in particular through the Ultraviolet Coronagraph Spectrometer (UVCS, Kohl et al. 1995), which offered the first opportunity to study the basic properties of the most intense EUV emission line profiles (e.g., the H I Ly $\alpha$ and O VI resonance doublet) as a function of heliocentric distance and latitude in the extended solar corona.

The ultraviolet emission lines present a rich and varied source of diagnostic information about the solar corona. The line intensity and profile for a given element depend on the electron density, the electron temperature, the density and kinetic temperature of the emitting ions, and on the outflow velocity. In the following text we report the physical parameters and their radial variations in a narrow, mid-latitude streamer structure, derived from observations carried out through a week in May 2004, during the declining phase of the solar activity cycle 23 . The empirical model describing the streamer characteristics presented in this paper is based on analysis of ultraviolet spectroscopic measurements in the extended corona obtained by UVCS and of white-light polarized brightness measurements from the LASCO (Large Angle Spectroscopic COronagraph) C2 coronagraph (Brueckner et al. 1995), both aboard the SOHO satellite. The values of these parameters are compared with the properties of streamers observed by SOHO instruments during the course of the solar cycle and/or at latitudes closer to the solar equator, with the aim of contributing to the ongoing campaign devoted 
Table 1. Summary of the UVCS observations.

\begin{tabular}{lcl}
\hline \hline Start date, Time $(\mathrm{UT})$ & PA $\left(^{\circ}\right)$ & Heliocentric distances $\left(R_{\odot}\right)$ \\
\hline May 17, 17:30 & 215 & $1.6,1.75,1.90,2.1$ \\
& 225 & $2.3,2.6$ \\
& 240 & $2.6,2.3$ \\
& 235 & $2.1,1.9,1.75,1.6$ \\
May 18, 17:45 & 235 & $2.5,2.75,3.0$ \\
May 19, 18:00 & 235 & $2.5,3.0,3.5$ \\
& 240 & 4.0 \\
& 240 & $3.5,3.0,2.5$ \\
May 20, 14:00 & 235 & $4.0,4.5$ \\
May 21, 19:40 & 235 & $2.5,3.0,3.5,4.0$ \\
May 22, 15:30 (O VI) & 235 & $4.5,5.0$ \\
May 22, 21:30 (Ly $\alpha)$ & 235 & $2.5,3.0,3.5,4.0,4.5,5.0$ \\
\hline
\end{tabular}

Note. The values of PA refer to the center of the entrance slit.

to the study of the physical characteristics of coronal streamers (see, e.g., Uzzo et al. 2006).

\section{Observations and data analysis}

The present work is based on spectral data collected during special observations carried out by UVCS/SOHO in 2004, from May 17 at 17:30 UT to May 23 at 06:30 UT. The measurements consist of radial and latitudinal scans of the extended corona above the southwest limb of the Sun, from 1.6 to $5.0 R_{\odot}$, in the $215^{\circ}-240^{\circ}$ range of position angle (PA, measured counterclockwise from solar north pole). On May 17 and 19 UVCS performed both radial and latitudinal scans, while only radial scans were carried out the other days. Details of the observation sequences are reported in Table 1. During the whole observation period, we monitored a mid-latitude quiescent streamer, located in the southwest quadrant. Note that the maximum values of the line intensities along the entrance slit correspond to PA's around $235^{\circ}$, at all the heights considered during the various days of observation. Only data pertaining to this region of the streamer have been analyzed in the present work.

Only intensities and profiles of the O VI resonance doublet lines were collected in the first 5 days, while the last day was devoted to acquiring intensities and profiles of both the O VI and Ly $\alpha$ lines, the latter through the redundant optical path of the O VI channel. The O VI data were taken with a spatial binning of 6 pixels, giving a spatial resolution of $42 \operatorname{arcsec}(7 \operatorname{arcsec}$ for each pixel) along a 40 arcmin entrance slit. The slit width was set equal to $50 \mu \mathrm{m}$ at $1.6 R_{\odot}$, and progressively increased up to $200 \mu \mathrm{m}$ at higher heliocentric distances in order to optimize both spectral resolution and photon flux. To meet the same requirement for Ly $\alpha$ observations, carried out only at or above $2.5 R_{\odot}$ (see Table 1), we adopted a spatial binning of 3 pixels and a slit width of $150 \mu \mathrm{m}$ at any height. The exposure time was $200 \mathrm{~s}$, in all cases, and the number of exposures was chosen for each position of the entrance slit so as to optimize the signal-to-noise ratio.

LASCO C2 pB images were also taken day by day from May 17 to May 22, 2004. The inversion of such pB data (see, e.g., Hayes et al. 2001) provided daily electron density maps relevant to the portion of extended corona observed in UV, in the 2.25 to $6 R_{\odot}$ range of heliocentric distance. We also determined the electron density $\left(N_{\mathrm{e}}\right)$ below $2.25 R_{\odot}$ by adopting the method described by Parenti et al. (2000), based on the dependence on $N_{\mathrm{e}}$ of the ratio of the radiative and collisional components of the O VI $1032 \AA$ line. This technique works under the condition of a nearly static plasma, as is the case of the examined streamer below $2.25 R_{\odot}$, so that the Doppler dimming effect on the radiatively excited component of the $\mathrm{O}$ VI lines can be considered negligible. Values of the ratio $\left(I_{\mathrm{rad}} / I_{\mathrm{col}}\right)_{1032}$ were derived from our UVCS data through the simple relationships between the intensities of the collisionally and radiatively excited components of the O VI $1032 \AA$ and $1037 \AA$ lines, i.e., $I_{\mathrm{col}}(1032) / I_{\mathrm{col}}(1037)=$ 2 and $I_{\text {rad }}(1032) / I_{\text {rad }}(1037)=4$ (see, also, Noci et al. 1987). Considering that the total intensity of each doublet line is given by $I=I_{\mathrm{rad}}+I_{\mathrm{col}}$, we obtain:

$\left(I_{\mathrm{rad}} / I_{\mathrm{col}}\right)_{1032}=\frac{2\left(I_{1032}-2 I_{1037}\right)}{4 I_{1037}-I_{1032}}$.

On May 18 and 19, in addition, the intensity and profile of the O VI resonance doublet lines on the solar disc were obtained by the spectrometer SUMER (Solar Ultraviolet Measurements of Emitted Radiation, Wilhelm et al. 1995), which observed two adjacent regions close to the southwest limb just below the observed streamer, approximately 5 arcmin wide in the $y$ direction. The SUMER data consist of high-resolution spectra centered on the O VI doublet at $1032 \AA$ and $1037 \AA$, observed using the $0.3^{\prime \prime} \times 120^{\prime \prime}$ slit. These spectra were taken at four different locations $\left(Y=-533^{\prime \prime},-433^{\prime \prime},-333^{\prime \prime},-233^{\prime \prime}\right)$ along the northsouth direction and at the same position along the eastwest direction $\left(X=750^{\prime \prime}\right)$. At each position along the northsouth direction, the observations were repeated 11 times with an exposure time of $30 \mathrm{~s}$. SUMER data were then decompressed, corrected from the flat-field image and geometrical distortions, and calibrated by using the standard software available as part of the SolarSoft package. SUMER data were averaged along the slit to increase the signal to noise ratio; the results for the same position along the northsouth direction were then averaged together, resulting in one single spectrum for each position along the northsouth direction. Gaussian line profiles were fitted from the resulting spectra, paying close attention to background removal.

These chromospheric spectral data were used to calculate the proper stray-light contribution coming from the solar disc to the O VI lines observed in corona and, together with disc intensities provided by other authors, to synthesize the radiatively excited component of the coronal lines expected from the streamer model (see below). Moreover, the disc intensity of the O VI $1032 \AA$ line was used in Eq. (8) of Parenti et al. (2000) to determine the electron density at heliocentric distances lower than $2.25 R_{\odot}$. As for the Ly $\alpha$ line, we adopted the average chomospheric profile reported by Gouttebroze et al. (1978), which agrees, within the uncertainties, with the full-disc Ly $\alpha$ intensities measured by the spectrometer SOLSTICE (SOLar STellar Irradiance Comparison Experiment) on the UARS satellite (see Woods et al. 2000) during the week of our observations.

Figure 1 shows a composite view of the extended solar corona and of the chromosphere, obtained on May 17, 2004. The $\mathrm{H} \alpha$ image of the solar disc, taken at the INAF-Catania Astrophysical Observatory, illustrates the conditions of the chromosphere on the first day of observation. Some instantaneous positions of the UVCS slit, superimposed on the LASCO C2 white-light image, are also reported. The presence of a group of filaments lying in a wide area under the streamer is evident. Some active regions are also present on the equatorial belt, but they do not seem to be connected to the streamer morphology. This fact should be kept in mind when interpreting the results.

Figure 2 reports the sequence of white-light LASCO C2 images, taken from May 18 to 22, 2004, together with some of the instaneous positions of the UVCS slit during the radial scans. 


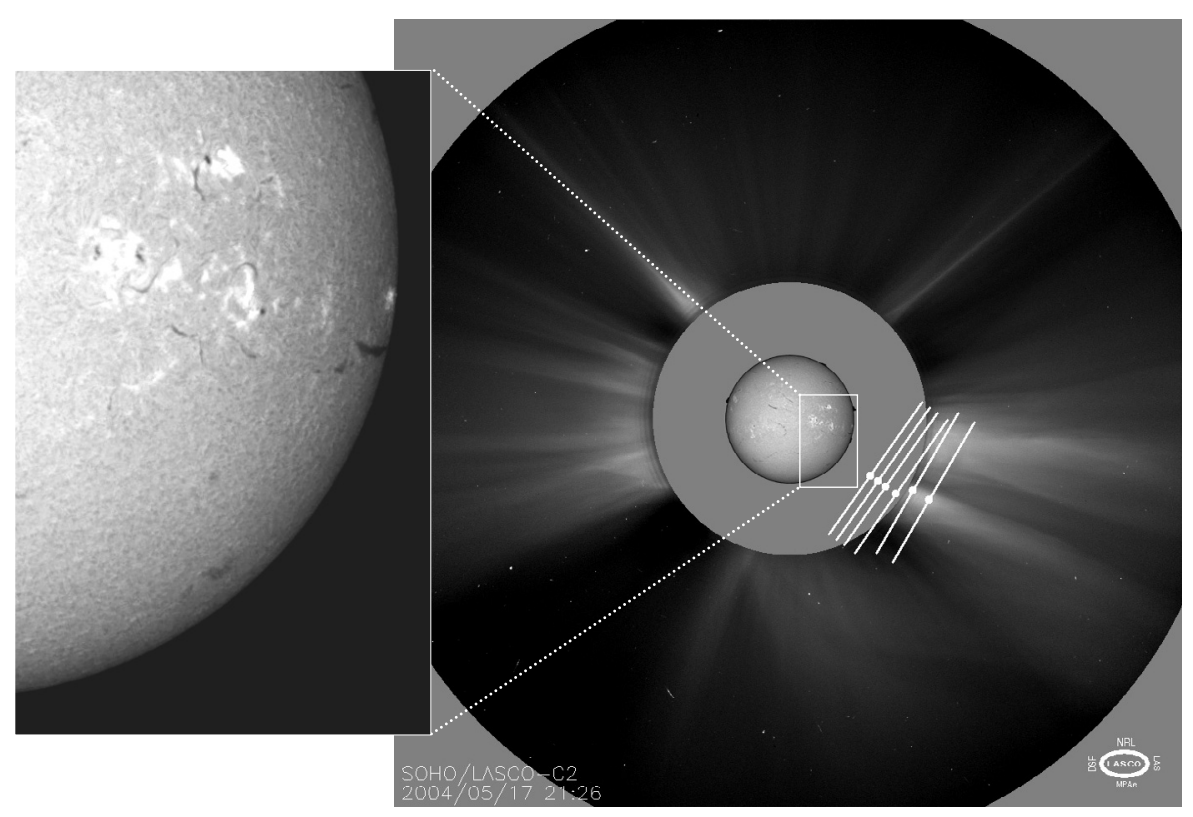

Fig. 1. Composite image obtained by combining the $\mathrm{H} \alpha$ image of the solar disc, taken at the INAF-Catania Astrophysical Observatory on May 17, 2004, and the LASCO C2 white-light image of the extended solar corona taken on the same day. Some instantaneous positions of the O VI channel entrance slit of UVCS (white segments) during the radial scan are shown superimposed on the LASCO image. The white dots mark the positions along the slit closest to the solar limb, to which the heliocentric distances and PA reported in Table 1 refer. A closer view of the disc region near the limb below the observed streamer, extracted from the $\mathrm{H} \alpha$ image, is also shown on the left.
It proves the different aspects of the streamer observed along the line of sight during the observation period as a consequence of the solar rotation. It is worth noting that on May 18, at 12:06 UT, a faint $\mathrm{CME}$ left the Sun along the $\mathrm{PA}=256^{\circ}$ direction, as reported in the SOHO LASCO CME catalog. However, it is classified as "very poor", and its influence on the streamer structure appears negligible, as can be deduced from LASCO C2 data and related movies.

The UVCS Data Analysis and processing Software (DAS, originally developed by C. Benna, A. van Ballegoijen, J. Raymond and S. Giordano) was used for flat-field correction, wavelength and radiometric calibration, removal of image distortion, and spectra extraction (Gardner et al. 1996; Kohl et al. 1997). Panasyuk's package (available from the Harvard-Smithsonian Center for Astrophysics website: cfa-www.harvard.edu/uvcs) was used to remove the stray light contribution to both the H I Ly $\alpha$ and O VI doublet lines, as well as the contribution from the interplanetary scattered Ly $\alpha$ line contained in the observed Ly $\alpha$ profile. Subtraction of the background and instrumental broadening correction were also performed before single Gaussian fits to the line profiles were obtained, in order to determine their intensity and $1 / e$ half width.

The values of the electron density, electron temperature, kinetic temperature, and outflow velocity of the emitting ions at different heliocentric distances in the streamer region centered around $\mathrm{PA}=235^{\circ}$ (hereafter indicated as central region of streamer) have been deduced by iteratively reproducing the above set of observational results as closely as possible, by means of a spectral synthesis code developed by Ventura \& Spadaro (1999). The code calculates both the radiatively and collisionally excited components of coronal lines from an adjustable model of the considered solar structure and average disc profiles of the chromospheric and inner corona exciting lines. It also includes the progressive depletion of the radiative components with increasing plasma outflow speed, known as the Doppler dimming effect (see, e.g., Kohl et al. 2006). We adopted an improved version of the code that takes possible anisotropies in the kinetic temperatures of the emitting ions into account, considering a bi-Maxwellian velocity distribution. As in Strachan et al. (2002) and Uzzo et al. (2006), we assumed that the bulk of the coronal emission from the streamer is contained within $\pm 0.2-0.3 R_{\odot}$ from the plane of the sky.

Atomic data, elemental abundances, and ionization balances are those reported in Ventura \& Spadaro (1999) and in papers quoted there. Note that the ionization balances for H I and O VI ions adopted in the present work were obtained with the assumption of ionization equilibrium. This appears to be fairly valid for low-velocity regimes, as can be expected to occur in streamer structures (cf., Spadaro \& Ventura 1994a,b). Moreover, the OVI line intensity ratio, suitable for obtaining information on the outflow speed inside a coronal structure, does not depend on the ionization balance of the ionic species (see Noci et al. 1987).

\section{Results and discussion}

\subsection{UVCS line intensities and profiles}

Figure 3 reports the total intensity of the O VI $1032 \AA$ line as a function of heliocentric distance along the central region of the streamer. The errors affecting the data points were computed as the standard deviation $( \pm 1 \sigma)$ of the Poisson photon-counting statistics. The O VI $1032 \AA$ intensity distribution, obtained as a combination of radial scans carried out in a time interval of 6 days (see Table 1), falls off with height by about three orders of magnitude. It is evident that the points relevant to May 18, 19, and 20 are systematically higher than those corresponding to the other observing days. The projection along the line of sight of different components of the streamer region, dragged westward by the solar rotation, might clearly account for the increase in the OVI emission, although we cannot rule out some effect of the CME that occurred on May 18. Even if this event did not appear to affect the streamer structure significantly, the possible presence of coronal material, left along the line of sight after the CME occurrence, could enhance the O VI $1032 \AA$ emission for a while.

Figure 4 reports the O VI line intensity ratio $I(1037 \AA)$ / $I(1032 \AA)$ as a function of the heliocentric distance along the central region of the streamer. This ratio was obtained by combining the values referring to the various observation days. The uncertainties in the line ratios have been deduced from the 

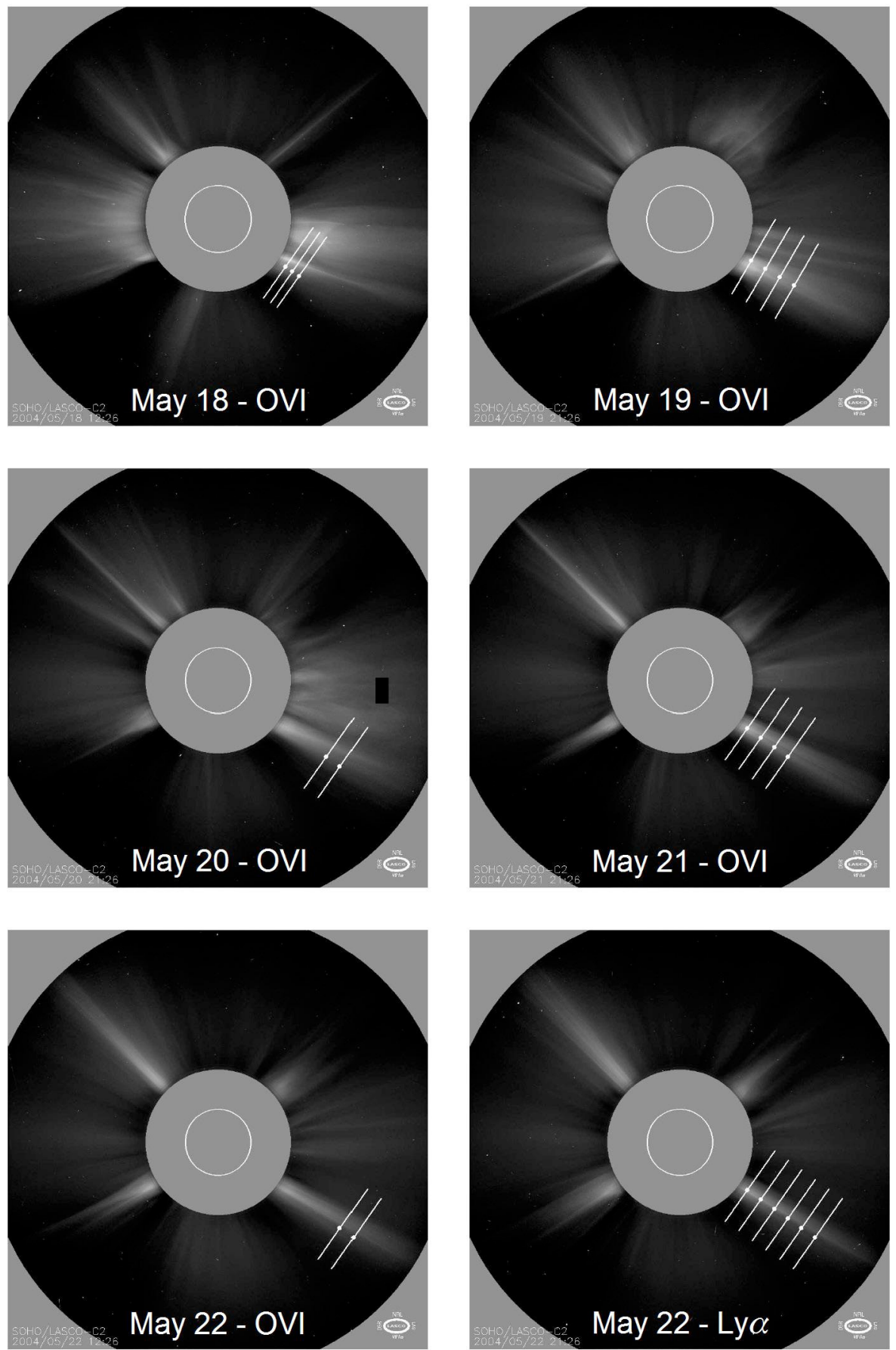

Fig. 2. LASCO C2 white-light images taken from May 18 to May 22, 2004, during the O VI scans and the Ly $\alpha$ scan, as indicated in each panel. Some instantaneous UVCS slit positions are also reported, superimposed on the LASCO images. statistical uncertainties in the relevant line intensities. Within $4.0 R_{\odot}$, the $\mathrm{O}$ VI intensity ratio is below or equal to 0.35 , with a flat minimum $\simeq 0.28$ between 2.5 and $3.5 R_{\odot}$. At greater heliocentric distances, the $\mathrm{O}$ VI intensity ratio increases up to nearly 0.5 , revealing a corresponding increase in the radial outflow velocity from values around $20 \mathrm{~km} \mathrm{~s}^{-1}$ to about $100 \mathrm{~km} \mathrm{~s}^{-1}$ (Noci et al. 1987; Noci \& Gavryuseva 2007). It is worth noting that the values of the line ratio referring to the days characterized by the enhancement in the O VI line intensities (see Fig. 3) are in excellent agreement with the behavior of the ratios derived from the data collected in the other days. Since CME eruptions typically involve flows of material with velocities well above $100 \mathrm{~km} \mathrm{~s}^{-1}$, implying line ratios significantly higher than 0.5 (Noci et al. 1987), this result appears to confirm the idea of a negligible influence of the observed CME on the dynamical structure of the monitored streamer.

Figure 5 shows the radial profile along the central region of the streamer of the O VI line-of-sight component of the kinetic temperature (hence, perpendicular to the solar radial direction, which is approximately the direction of the magnetic field), deduced from the $1 / e$ half widths of the O VI $1032 \AA$ spectral line. The uncertainties in the kinetic temperature have been estimated from the uncertainties affecting the line widths, through the error propagation statistical treatment (see Zangrilli et al. 1999). The corresponding $\sigma$ values are plotted as error bars in Fig. 5. The most evident feature of this radial profile is its quickly increasing trend, from $\sim 2-3 \mathrm{MK}$ to $\sim 20 \mathrm{MK}$, in the range of heliocentric 


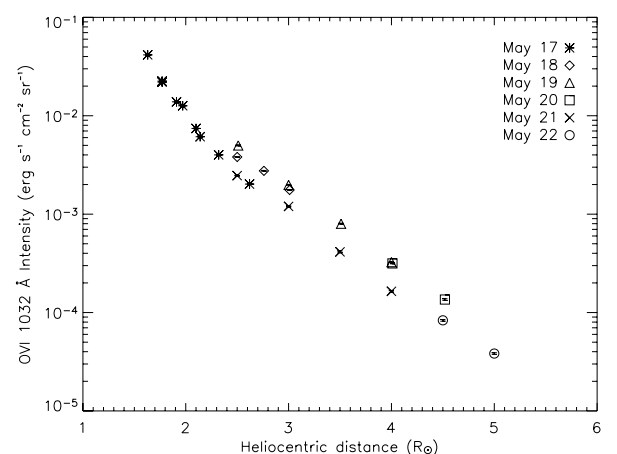

Fig. 3. O VI $1032 \AA$ line-integrated intensities reported as a function of the heliocentric distance along the central region of the mid-latitude streamer observed from May 17 to May 22, 2004. Different symbols refer to different observation days. The error bars, determined as described in the text, are smaller than the symbol size for all the data points.

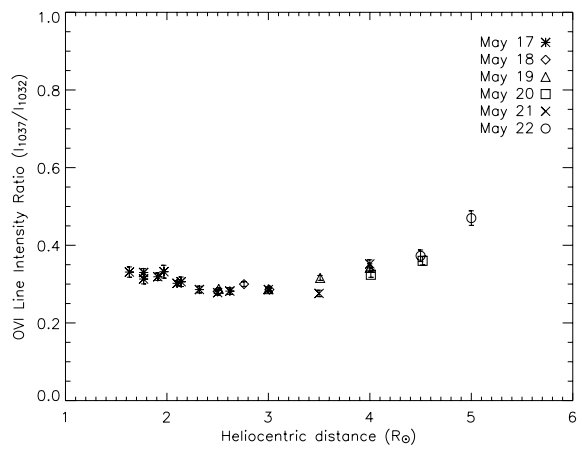

Fig. 4. As in Fig. 3, for the O VI (1037 $\AA / 1032 \AA)$ line intensity ratio. The error bars, determined as described in the text, are smaller than the symbol size for many of the data points.

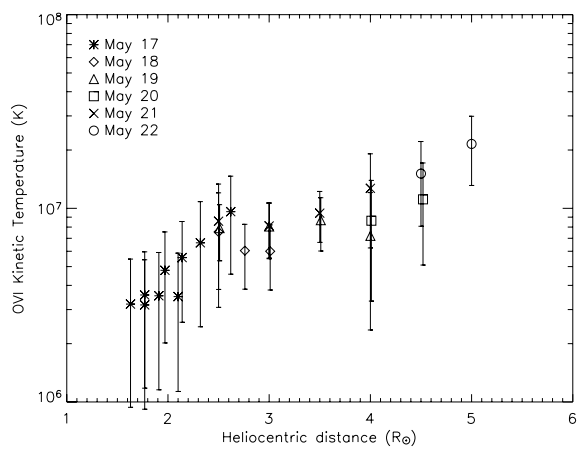

Fig. 5. As in Fig. 3, for the O VI perpendicular kinetic temperature, i.e., along the line of sight. See the text for a description of the error bar determination.

distance considered. This suggests that some kind of heating might be taking place within the streamer.

Figure 6 reports the total intensity of the $\mathrm{HI} \operatorname{Ly} \alpha$ line as a function of heliocentric distance along the central region of the streamer, as determined by the radial scan carried out in the 2.5-5.0 $R_{\odot}$ range, from 21:30 UT on May 22 to 6:30 UT on May 23 (see Table 1). The errors affecting the data points were computed as in the case of O VI lines. The Ly $\alpha$ intensity radial profile exhibits a shallower decrease than that of the O VI line observed on May 21 and 22: in the same range of heights, we notice a decrease slightly larger than one order of magnitude for the former and about two orders of magnitude for the latter.

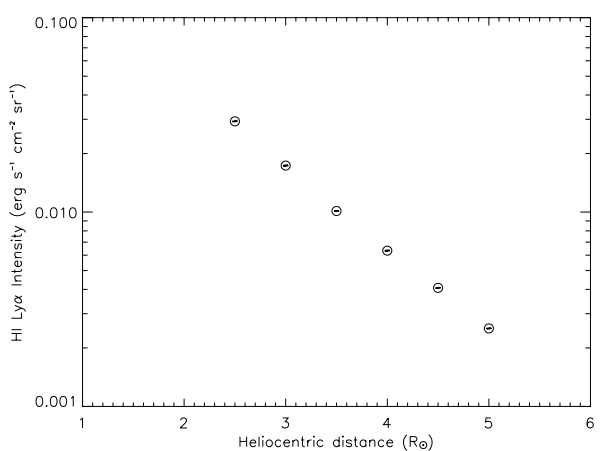

Fig. 6. Line-integrated intensities of the H I Ly $\alpha$ line as a function of heliocentric distance along the central region of the streamer, obtained on May 22-23, 2004. The error bars, determined as described in the text, are smaller than the symbol size for all the data points.

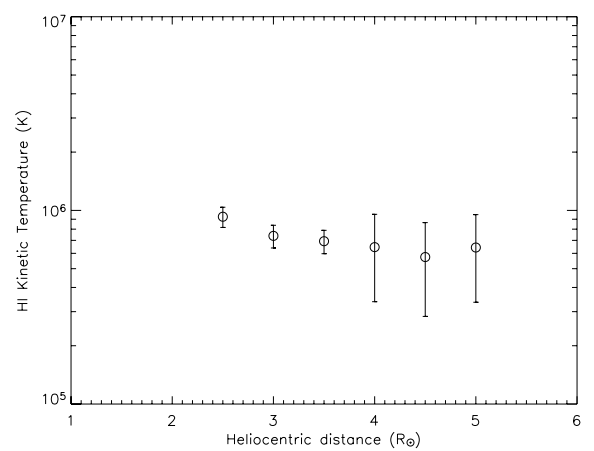

Fig. 7. As in Fig. 6, for the H I perpendicular kinetic temperature, i.e., along the line of sight. See the text for a description of the error bar determination.

Besides the possible differences due to the solar rotation, it is worth noting that, as discussed in previous papers (e.g., Ventura et al. 2005), the two lines fall off differently with height because Ly $\alpha$ emission is dominated by resonance scattering, proportional to the density, while the O VI line also has a collisionally excited component, which depends on density squared.

Figure 7 shows the radial profile of the $\mathrm{HI}$ perpendicular kinetic temperature derived as in the case of the O VI ions, together with their related uncertainties. Due to the high rate of charge exchange between protons and neutral hydrogen, we can use the Ly $\alpha$ line widths as a proxy for the velocity distribution of protons along the line of sight (e.g., Withbroe et al. 1982; Allen et al. 1998; Vásquez et al. 2003). The small decrease in the H I perpendicular kinetic temperature, by about a factor 1.4 in the 2.5-5.0 $R_{\odot}$ range, and the values always below or equal to $1 \mathrm{MK}$ are the main characteristics of a behavior opposite that of the $\mathrm{O}$ VI ions (see Fig. 5). This different behavior has already been described in the literature (e.g., Strachan et al. 2002; Ventura et al. 2005; Uzzo et al. 2006) and hints at mechanisms that preferentially heat $\mathrm{O}$ VI ions more than protons.

\subsection{Diagnostics of physical parameters}

The above set of observational results were used as constraints to derive the plasma conditions in the monitored streamer structure. In fact, the Ly $\alpha$ and O VI resonance-doublet line intensities and profiles depend on the electron density, the electron temperature, the density and kinetic temperature of the emitting ions, and the outflow velocity, so that the radial profiles of these physical parameters along the central region of the streamer can be 


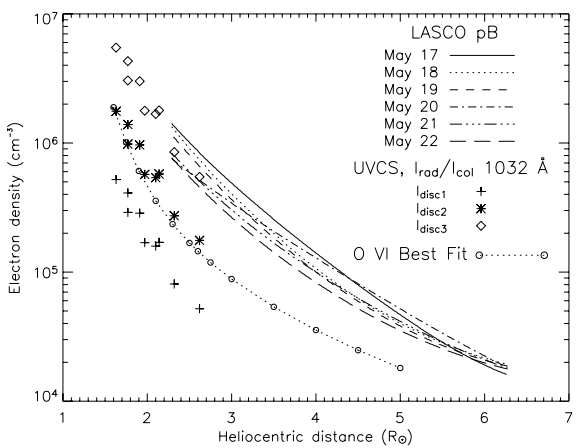

Fig. 8. Electron densities derived from the inversion of LASCO C2 $\mathrm{pB}$ data relevant to the different days of observation, represented as a function of heliocentric distance by different curves. Also reported are the densities obtained from UVCS line intensities below $2.6 R_{\odot}$. Different symbols refer to values obtained adopting different O VI $1032 \AA$ disc intensity inputs, namely $I_{\text {disc } 1}=116, I_{\text {disc } 2}=305$, $I_{\text {disc3 }}=610 \mathrm{erg} \mathrm{cm}^{-2} \mathrm{sr}^{-1} \mathrm{~s}^{-1}$. Finally, the open circle-dotted line represents the electron density profile giving the best agreement between the synthesized and the observed O VI line emission.

determined after iteratively reproducing the spectroscopic data reported in the previous subsection as closely as possible by means of the synthesis code quoted in Sect. 2 (see, e.g., Ventura \& Spadaro 1999).

The iterative procedure starts from an initial estimate of the above plasma parameters, deduced from the UV line intensities and profiles, as well as white-light $\mathrm{pB}$ data, collected during the whole observation period (see Sect. 2 and, also, cf. Kohl et al. 1997). The spectral characteristics of the emission lines synthesized by this initial model are compared with the measured spectroscopic observables. Then the plasma parameters are iteratively re-adjusted under the guidance provided by the repeated comparison of the synthetic observables with the data, until an optimal consistency with the observations is obtained (cf., e.g., Kohl et al. 2006). The agreement of the emission-line intensities, profiles, and intensity ratios synthesized from the resulting empirical model of the streamer with the spectral data reported in Figs. 3-7 appears remarkable, well within the data uncertainties, particularly in the case of the $\mathrm{O}$ VI line intensity ratio.

Figure 8 shows the radial profiles of the electron density along the central region of the mid-latitude streamer monitored during our week of observations, derived from the inversion of LASCO C2 pB data relevant to the different days of observation, together with the density values obtained at lower heliocentric distances from the UVCS data collected on May 17 (see Table 1), adopting the method proposed by Parenti et al. (2000).

The various density profiles obtained by the LASCO C2 $\mathrm{pB}$ data inversion overlap at heliocentric distances greater than $5.5 R_{\odot}$, while they fan out progressively at lower heights, even if the largest differences among the various curves are always within a factor of two. This is reasonable, due to the changing shape and aspect of the streamer over the observation days. The Ly $\alpha$ intensities and profiles synthesized by adopting the electron density values relevant to May 22, also taking into account the Doppler dimming effect due to the outflow velocity, reproduce the spectral data reported in Figs. 6 and 7 quite well.

The electron densities computed by Eq. (8) of Parenti et al. (2000) were obtained assuming an electron temperature equal to $1.1 \times 10^{6} \mathrm{~K}$ (Fineschi et al. 1998) over all the 1.6-2.6 $R_{\odot}$ range of heliocentric distances. Different symbols refer to different values of the O VI $1032 \AA$ disc intensity adopted in the relationship from which densities have been derived, respectively 116, 305, and $610 \mathrm{erg} \mathrm{cm}^{-2} \mathrm{sr}^{-1} \mathrm{~s}^{-1}$. The first intensity value corresponds to the average of the SUMER disc measurements carried out on May 18 and 19. The resulting density values are more than one order of magnitude lower than those deduced by LASCO C2 pB data at 2.3 and $2.6 R_{\odot}$ and hardly compatible with an ideal extrapolation of LASCO density curves at lower heights. The second value of the O VI disc intensity is the quiet-Sun contribution listed in Vernazza \& Reeves (1978), while the third one was adopted in order to get densities satisfactorily matching those derived from LASCO C2 pB data. An intensity value about five times higher than measured by SUMER could be considered questionable at first glance. However, the lack of simultaneous measurements of disc intensities is a source of uncertainty whose influence is hardly quantifiable, as we do not really know how large a variability we can expect in the O VI $1032 \AA$ disc intensities. Moreover, SUMER observations are relevant to a limited quiet region close to the limb below the observed streamer, while a significantly higher contribution to the disc radiation could also come from some of the active regions near the equator visible in the $\mathrm{H} \alpha$ image of May 17 (see Fig. 1), and still present on the following days (see, for instance, Morgan \& Habbal 2005).

Also reported in Fig. 8 is the electron density profile giving the best synthesis of the observed O VI emission line intensities, profiles, and intensity ratios. These density values are systematically lower than those derived by LASCO C 2 pB data inversion, in some cases even a factor of five lower. At low heliocentric distances, they are in good agreement with the densities obtained by the method of Parenti et al. (2000), with an O VI disc intensity input equal to the quiet-Sun value reported by Vernazza \& Reeves (1978).

The observed values of the resonance-doublet line ratio $I(1037 \AA) / I(1032 \AA)$ strongly indicate that the densities derived from LASCO data are too high to explain the streamer emission in the O VI lines. They result in synthetic O VI intensity ratios systematically close to 0.5 , corresponding to resonancedoublet line emission dominated by the collisionally excited components (Noci et al. 1987), even at heliocentric distances below $4.0 R_{\odot}$, where the observed curve exhibits lower values and the characteristic shape discussed by Noci et al. (1987) and Noci \& Gavryuseva (2007). This holds even if we adopt the highest $\mathrm{O}$ VI disc intensities listed above in the calculation of the radiatively excited components of the coronal lines. On the other hand, the use of the lowest density values reported in Fig. 8 results in synthetic $\mathrm{O}$ VI intensity ratios almost close to 0.25 , the ratio of the radiatively excited components of the lines (Noci et al. 1987), already below $2.5 R_{\odot}$, whatever the O VI disc intensities adopted among those listed above. Hence we also consider these densities unsuitable for explaining the observed O VI emission.

The striking differences among the electron density values obtained by the LASCO C2 pB data inversion, which nicely reproduce $\mathrm{HI} \operatorname{Ly} \alpha$ observations, and those adopted to fit the O VI resonance doublet data (see Fig. 8) deserve a further and more thorough analysis, also considering that such differences are indeed larger than the estimated density uncertainties, which are well below a factor of two (cf. Hayes et al. 2001; Strachan et al. 2002). We need to take into account that the $\mathrm{pB}$ inversion assumes axisymmetry, so that the derived density represents a structure with significant depth along the line of sight. If we are looking at a narrow structure away from the plane of the sky, and this is conceivable for the mid-latitude streamer examined here, it might contribute little brightness compared to the background 


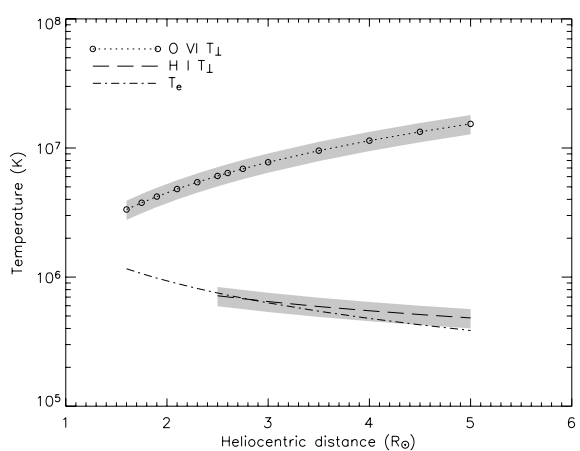

Fig. 9. Radial profiles of the OVI and H I kinetic temperatures in the direction perpendicular to the magnetic field $\left(T_{\perp}\right)$, according to the derived empirical model. Grey bands represent the average ranges of uncertainty affecting these temperature curves. Also reported is the radial profile of the electron temperature $\left(T_{\mathrm{e}}\right)$, determined as described in the text.

corona. However, we can get a much higher density for the region in the plane of the sky corresponding to the projection of the considered structure, because of the high contribution from the background corona. This could be the case for the Ly $\alpha$ and $\mathrm{O}$ VI lines originating in the streamer investigated in this work: Ly $\alpha$, and most of white-light radiation, could originate in a wider and denser region along our line of sight, while the O VI emitting ions could be confined over a smaller volume. This could explain why lower densities are required to fit the O VI resonance doublet data.

Once the electron density had been derived, we deduced the electron temperature $\left(T_{\mathrm{e}}\right)$ profile along the central region of the streamer according to the method described by Gibson et al. (1999), which calculates temperatures from radial power-law fits to the combined density data set, assuming that the streamer plasma is in radial hydrostatic equilibrium. This implies that there are no significant dynamic forces and that thermal pressure is balanced by gravity. This can reasonably apply to our case, because the wind velocity may be considered as a minor term in force balance. Actually, the radial outflow velocities determined in this study reach values of about $100 \mathrm{~km} \mathrm{~s}^{-1}$ at heights around $5 R_{\odot}$, while the thermal velocities of electrons corresponding to the temperatures found in the examined streamer are significantly higher than this value everywhere (see also the discussion in Gibson et al. 1999). The computations were carried out using both the density profile deduced from LASCO C2 $\mathrm{pB}$ data inversion and the one giving the best fit of the O VI observations. The differences between the two sets of $T_{\mathrm{e}}$ data so determined can be considered negligible, being around $10 \%$. We finally adopted the $T_{\mathrm{e}}$ curve relevant to the density profile deduced from LASCO data.

The empirical radial profile of $T_{\mathrm{e}}$ is reported in Fig. 9, together with the profiles of the component of the kinetic temperature in the direction perpendicular to the magnetic field $\left(T_{\perp}\right)$ derived for $\mathrm{HI}$ atoms (and protons) and $\mathrm{O}$ VI ions. The average uncertainty ranges pertaining to the empirical kinetic temperature curves are also reported in the figure: they were derived in terms of the lowest and highest temperature values that are still consistent with the observed line intensities, profiles, and $\mathrm{O}$ VI intensity ratios, within the observational uncertainties. Both the radial profiles of $T_{\mathrm{e}}$ and $\mathrm{H} \mathrm{I} T_{\perp}$ exhibit a slight decrease, practically overlapping between 2.5 and $3.3 R_{\odot}$ and then only slightly diverging at greater heliocentric distances. This agrees with the hypothesis of thermal equilibrium between electrons and hydrogen atoms (protons), suggested on the basis of the high-density

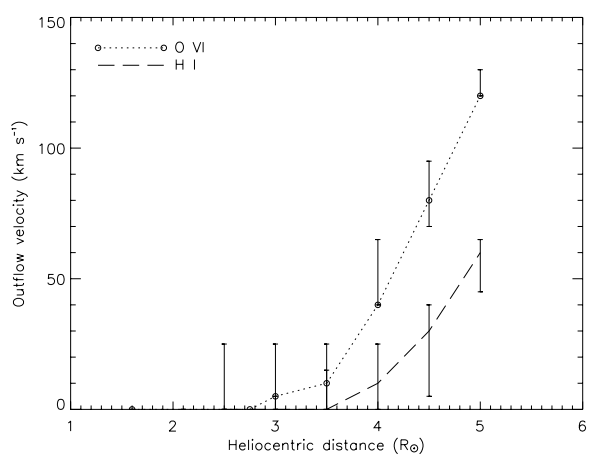

Fig. 10. Radial profiles of the outflow velocity along the streamer axis for the HI and O VI ions, according to the derived empirical model. The error bars refer to the uncertainties estimated at the various heliocentric distances as described in the text.

and low-outflow conditions characterizing streamers, expecially at low heights (cf., Raymond et al. 1997; Vásquez et al. 2003). The $\mathrm{O}$ VI $T_{\perp}$, already as high as $3 \mathrm{MK}$ at $1.6 R_{\odot}$, is characterized by a significant increase with height, up to nearly $15 \mathrm{MK}$, hence exhibiting much higher values than the corresponding hydrogen temperature.

As for the components of the H I and O VI kinetic temperature parallel to the magnetic field lines $\left(T_{\|}\right)$, not plotted in Fig. 9, we adopted different values in the development of the empirical model, so as to consider both isotropic $\left(T_{\|}=T_{\perp}\right)$ and anisotropic conditions with $T_{\|}=T_{\perp} / 2, T_{\perp} / 1.3$, respectively. The last value, simultaneously adopted for both neutral hydrogen and oxygen ions, seems to give the best reproduction of the observed Ly $\alpha$ and O VI spectral data, even if we cannot rule out the possibility of isotropic temperatures, considering the uncertainties characterizing the empirical curves plotted in the figure. On the other hand, such mildly anisotropic velocity distributions of the emitting ions are almost similar to those found by Frazin et al. (2003) in the stalk of an equatorial streamer observed at solar activity minimum.

The values of the outflow velocity along the central region of the streamer for both the HI and O VI ions were initially set equal to zero. This choice is reasonably supported by many pieces of observational evidence, suggesting that streamer axes are characterized by low-velocity outflows (e.g., Strachan et al. 2002; Uzzo et al. 2006). Subsequently, the ion speeds were progressively increased in order to match all the spectroscopic observations, until the empirical radial profiles of the $\mathrm{HI}$ and $\mathrm{O}$ VI outflow velocity reported in Fig. 10 were obtained. The error bars were deduced for each point by a procedure similar to that adopted for estimating the temperature uncertainties, and they result in about $\pm 20 \mathrm{~km} \mathrm{~s}^{-1}$. Evidence of a significant deviation from static conditions only appears at about 3.5-4.0 $R_{\odot}$, for both $\mathrm{HI}$ and $\mathrm{O}$ VI ions, with a progressive steepening of the outflow velocity radial profiles at greater heliocentric distances. However, the outflow velocity of coronal O VI ions at lower heights in the central region of the streamer might be underestimated in our analysis, which adopted the quiet Sun O VI line disc intensities reported by Vernazza \& Reeves (1978) to perfom the synthesis of O VI emission in the streamer. Even if this approach gives a very consistent agreement with the observations, a non-negligible O VI outflow below $3.0 R_{\odot}$ could result from the analysis of the observed line intensity ratio, when the possible influence of the active region sunspots present on the solar disc during the week of observation on the intensity of the 
radiative scattering component of the coronal $\mathrm{O}$ VI lines is also considered, as discussed by Morgan \& Habbal (2005).

Above $4 R_{\odot}$, the $\mathrm{O}$ VI outflow velocity increases more rapidly than does that of $\mathrm{HI}$, indicating that the former ions may flow as much as 1.5-2 times faster than the latter ones, reaching a speed of $120 \mathrm{~km} \mathrm{~s}^{-1}$ at $5 R_{\odot}$. The faster O VI outflow velocity, together with the $\mathrm{O}$ VI kinetic temperatures that are much higher than the hydrogen temperatures (see above), suggest that the absorption of Alfvén waves at the ion cyclotron frequency, as proposed by several authors (e.g., Li et al. 1997, 1999; Tu \& Marsch 1997; Cranmer et al. 1999a, 1999b; Hu \& Habbal 1999) to explain both the high kinetic temperatures and the high ion outflow velocities observed in coronal holes, might also occur inside streamers, at least above a certain height.

\section{Conclusions}

The plasma parameters empirically determined at several heights in the central region of a narrow, mid-latitude streamer structure observed along different lines of sight through a week during the declining phase of the solar activity cycle 23 , exhibit the following overall behavior.

The electron densities derived from the inversion of LASCO C2 pB data range from $\sim 5 \times 10^{6} \mathrm{~cm}^{-3}$ to $\sim 4 \times 10^{4} \mathrm{~cm}^{-3}$ in the 1.6-5 $R_{\odot}$ interval of heliocentric distance, without considerable differences among the curves relevant to the different days of observation. These densities appear to reproduce the Ly $\alpha$ data collected on May 22-23 quite well, while they are too high to explain the streamer emission in the O VI resonance doublet lines. To fit these data, electron densities are required that are a factor of three - four lower, so that they range from $\sim 2 \times$ $10^{6} \mathrm{~cm}^{-3}$ (at $1.6 R_{\odot}$ ) to $\sim 1.5 \times 10^{4} \mathrm{~cm}^{-3}$ (at $5 R_{\odot}$ ). Taking the axisymmetry assumed in the $\mathrm{pB}$ inversion into account, the above differences could result from a wider and denser region along the line of sight contributing to the white-light and Ly $\alpha$ radiation, while the OVI ions are probably confined over a smaller volume, away from the plane of the sky. On the other hand, Antonucci et al. (2005) report that the electron densities derived from the analysis of $\mathrm{O}$ VI doublet lines are generally lower than those inferred from the visible light observations.

The electron temperature profile calculated from radial power-law fits to the combined density data set exhibits a slight decrease, with values ranging from $\sim 1.2 \mathrm{MK}$ to $\sim 0.4 \mathrm{MK}$ in the 1.6-5 $R_{\odot}$ interval of heliocentric distance. The behavior of the component of the $\mathrm{H}$ I kinetic temperature in the direction perpendicular to the magnetic field is very similar, with only slightly higher values above $3.5 R_{\odot}$. The corresponding component of the OVI kinetic temperature is characterized by a significant increase with height, from about $3 \mathrm{MK}$ at $1.6 R_{\odot}$ up to nearly $15 \mathrm{MK}$ at $5 R_{\odot}$. A mild anisotropy is noticed in the velocity distributions of the emitting ions, with the components of the $\mathrm{HI}$ and $\mathrm{O}$ VI kinetic temperature parallel to the magnetic field a factor of 1.3 smaller than the respective perpendicular components.

The outflow velocities appear negligible up to $3 R_{\odot}$, whereas evidence of a significant deviation from static conditions appears at 3.5-4.0 $R_{\odot}$ for both $\mathrm{H} \mathrm{I}$ and $\mathrm{O}$ VI ions, suggesting that the transition from closed to open magnetic field regions (the so-called streamer cusp) might occur within this range of heliocentric distance. Then the ion outflow velocities increase outward, reaching values of $60 \mathrm{~km} \mathrm{~s}^{-1}(\mathrm{HI})$ and $120 \mathrm{~km} \mathrm{~s}^{-1}(\mathrm{O} \mathrm{VI})$ at $5 R_{\odot}$. It is worth noting that the $\mathrm{HI}$ outflow velocities obtained in this work are, according to our knowledge of the literature, the first ones determined inside a streamer structure. We also note that the faster O VI outflow velocity, together with the O VI kinetic

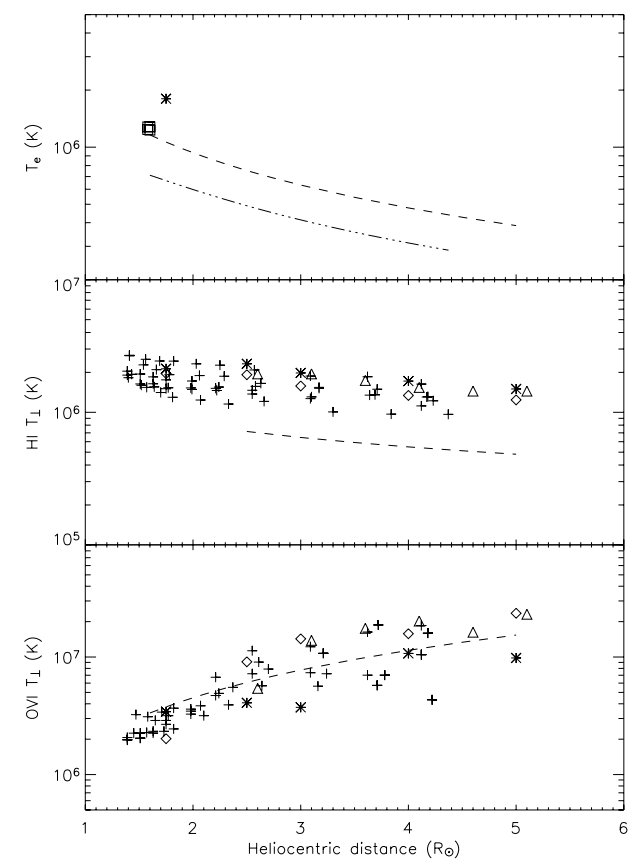

Fig. 11. $T_{\mathrm{e}}$ (top), $\mathrm{H} \mathrm{I} T_{\perp}$ (middle), and O VI $T_{\perp}$ (bottom) radial profiles derived in the streamer examined here (dashed line) and in those investigated by Gibson et al. (1999) - dash-dot-dot-dot line, Parenti et al. (2000) - squares, Strachan et al. (2002) - diamonds, Frazin et al. (2003) - triangles, Ventura et al. (2005) - crosses, Uzzo et al. (2006) asterisks.

temperatures that are much higher than the $\mathrm{H}$ I ones, suggest that the absorption of Alfvén waves at the ion cyclotron frequency might also occur inside streamers, at least above a certain height.

The physical properties summarized above can be compared with those of some other streamers described in the literature, observed during different phases of the solar cycle and/or at different latitudes. This comparison may contribute to the ongoing campaign devoted to studying the physical characteristics of coronal streamers and investigating whether structures grouped on the basis of their morphology, latitude, and solarcycle phase appearance also share common physical conditions (see, e.g., Parenti et al. 2000; Uzzo et al. 2006). It is worth noting that the quiescent streamer reported by Uzzo et al. (2006) was at a similar latitude to the one investigated here and was observed in April 2003 while the solar activity was still decreasing. Conversely, the equatorial streamers of Gibson et al. (1999) and Strachan et al. (2002), the equatorial and mid-latitude active region streamers of Parenti et al. (2000) and the depleted-core equatorial streamer of Frazin et al. (2003) were observed during a minimum phase of the solar activity. The results presented by Ventura et al. (2005) concern a collection of streamer observations performed at different latitudes in 1997 and 2000, hence near solar minimum and maximum respectively.

The densities derived from our LASCO data are in general agreement with those reported by the authors quoted above, except for Strachan et al. (2002) and Uzzo et al. (2006), who find values at least a factor of two higher. The densities fitting our O VI data are only similar to those obtained by Ventura et al. (2005) in some mid-latitude streamers at solar minimum, but considerably lower than the values reported in other investigations.

The comparisons of $T_{\mathrm{e}}, \mathrm{HI} T_{\perp}$ and $\mathrm{O}$ VI $T_{\perp}$ radial profiles derived in this work with the corresponding ones obtained in some other streamers described in the literature are summarized 
in Fig. 11. Significant differences are noticed among our electron temperature and $\mathrm{HI}$ kinetic temperature values and those reported by the majority of the other authors. As for the O VI kinetic temperatures, conversely, the agreement is generally better.

The outflow velocities derived for the O VI ions are consistent with the results concerning the other examined streamers quoted above. No significant radial outflows appear below $3 R_{\odot}$, while the transition towards non-static conditions occurs within $3.5-4 R_{\odot}$ in all the streamer structures considered. This behavior has also been noticed in some streamers investigated by Antonucci et al. (2005), observed near solar minimum. Above $4 R_{\odot}$ all the streamers exhibit a sharp increase in the outflow velocity, up to values around $100 \mathrm{~km} \mathrm{~s}^{-1}$ at $5 R_{\odot}$. This increase is steeper in the structure examined here than in those described by Strachan et al. (2002) and Uzzo et al. (2006).

Finally, we computed the 1 AU scaled particle-flux density from the densities and outflow velocities determined at $5 R_{\odot}$, assuming a pure radial expansion factor (see, for instance, Strachan et al. 2002). We find $\sim 1.3 \times 10^{8} \mathrm{~cm}^{-2} \mathrm{~s}^{-1}$. This is a factor of three lower than the in situ measurements for the particle flux density of the slow solar wind (e.g., Gosling 1997; Phillips et al. 1995), and even lower than the value of $5.4 \times 10^{8} \mathrm{~cm}^{-2} \mathrm{~s}^{-1}$, found by Strachan et al. (2002) in an equatorial streamer at solar minimum.

Acknowledgements. The authors wish to thank Y.-K. Ko for her assistance during UVCS/SOHO observations carried out at NASA GSFC, and R. Suleiman for his help in the preliminary reduction of the UVCS raw data. They also thank S. R. Habbal for her comments and suggestions, which led to a much sounder version of the manuscript. This work was supported in part by the Agenzia Spaziale Italiana (contracts ASI I/R/84/02 and I/035/05/0). The work of E. Landi is supported by the NNG06EA14I, NNH06CD24C and other NASA grants.

\section{References}

Allen, L. A., Habbal, S. R., \& Hu, Y. Q. 1998, JGR, 103, 6551

Antonucci, E., Abbo, L., \& Dodero, M. A. 2005, A\&A, 435, 699

Brueckner, G. E., Howard, R. A., Koomen, M. J., et al. 1995, Sol. Phys., 162, 357

Cranmer, S. R., Field, G. B., \& Kohl, J. L. 1999a, ApJ, 518, 937

Cranmer, S. R., Kohl, J. L., Noci, G. C., et al. 1999b, ApJ, 511, 481

Domingo, V., Fleck, B., \& Poland, A. I. 1995, Sol. Phys., 162, 1

Fineschi, S., Gardner, L. D., Kohl, J. L., Romoli, M., \& Noci, G. C. 1998, Proc. SPIE, 3443, 67
Frazin, R. A., Cranmer, S. R., \& Kohl, J. L. 2003, ApJ, 597, 1145 Gardner, L. D., Kohl, J. L., Daigneau, P. S., et al. 1996, Proc. SPIE, 2831, 2 Gibson, S. E., Fludra, A., Bagenal, F., et al. 1999, JGR, 104, 9691 Gosling, J. T. 1997, Robotic Exploration Close to the Sun, ed. S. R. Habbal (Woodbury: AIP), AIP Conf. Proc., 385, 17

Gouttebroze, P., Lemaire, P., Vial, J. C., \& Artzner, G. 1978, ApJ, 225, 655

Habbal, S. R., Woo, R., Fineschi, S., et al. 1997, ApJ, 489, L103

Hayes, A. P., Vourlidas, A., \& Howard, R. A. 2001, ApJ, 548, 1081

Hu, Y. Q., \& Habbal, S. R. 1999, JGR, 104, 17,045

Kohl, J. L., Esser, R., Gardner, L. D., et al. 1995, Sol. Phys., 162, 313

Kohl, J. L., Noci, G., Antonucci, E., et al. 1997, Sol. Phys., 175, 613

Kohl, J. L., Noci, G., Cranmer, S. R., \& Raymond, J. C. 2006, A\&AR, 13, 31

Lemaire, P., Wilhelm, K., Curdt, W., et al. 1997, Sol. Phys., 170, 105

Li, X., Esser, R., Habbal, S. R., \& Hu, Y.-Q. 1997, JGR, 102, 17419

Li, X., Habbal, S. R., Hollweg, J. V., \& Esser, R. 1999, JGR, 104, 2521

Low, B. C. 2001, JGR, 106, 25141

Marsch, E. 1997, in Fifth SOHO Workshop, The Corona and Solar Wind near Minimum Activity, ed. A. Wilson (Noordwijk: ESA), ESA-SP 404, 135

Morgan, H., \& Habbal, S. R. 2005, ApJ, 630, L189

Noci, G., \& Gavryuseva, E. 2007, ApJ, 658, L63

Noci, G., Kohl, J. L., \& Withbroe, G. L. 1987, ApJ, 315, 706

Noci, G., Kohl, J. L., Antonucci, E., et al. 1997, in Fifth SOHO Workshop, The Corona and Solar Wind near Minimum Activity, ed. A. Wilson (Noordwijk: ESA), ESA-SP 404, 75

Parenti, S., Bromage, B. J. I., Poletto, G., et al. 2000, A\&A, 363, 800

Phillips, J. L., Bame, S. J., Barnes, A., et al. 1995, GRL, 22, 3301

Raymond, J. C., Kohl, J. L., Noci, G., et al. 1997, Sol. Phys., 175, 645

Spadaro, D., \& Ventura, R. 1993, A\&A, 276, 571

Spadaro, D., \& Ventura, R. 1994a, A\&A, 281, 245

Spadaro, D., \& Ventura, R. 1994b, A\&A, 289, 279

Spadaro, D., \& Ventura, R. 1996, A\&AS, 115, 531

Strachan, L., Suleiman, R., Panasyuk, A. V., Biesecker, D. A., \& Kohl, J. L. 2002, ApJ, 571, 1008

Tu, C.-Y., \& Marsch, E. 1997, Sol. Phys., 171, 363

Uzzo, M., Strachan, L., Vourlidas, A., Ko, Y.-K., \& Raymond, J. C. 2006, ApJ, 645,720

Vásquez, A. M., van Ballegooijen, A. A., \& Raymond, J. C. 2003, ApJ, 598, 1361

Ventura, R., \& Spadaro, D. 1999, A\&A, 341, 264

Ventura, R., Spadaro, D., Cimino, G., \& Romoli, M. 2005, A\&A, 430, 701

Vernazza, J. E., \& Reeves, E. M. 1978, ApJS, 37, 485

Wilhelm, K., Curdt, W., Marsch, E., et al. 1995, Sol. Phys., 162, 189

Wilhelm, K., Lemaire, P., Curdt, W., et al. 1997, Sol. Phys., 170, 75

Withbroe, G. L., Kohl, J. L., Weiser, H., \& Munro, R. H. 1982, Space Sci. Rev., 33, 17

Woo, R., \& Martin, J. 1997, GRL, 24, 2535

Woods, T. N., Tobiska, W. K., Rottman, G. J., \& Worden, J. R. 2000, JGR, 105, 27195

Zangrilli, L., Nicolosi, P., Poletto, G., et al. 1999, A\&A, 342, 592 